\title{
Tiny masking locked nucleic acids effectively bind to mRNA and inhibit binding of microRNAs in relation to thermodynamic stability
}

\author{
KAZUYOSHI MURAKAMI $^{1,2}$ and MAKOTO MIYAGISHI ${ }^{2}$ \\ ${ }^{1}$ Graduate School of Frontier Sciences, University of Tokyo, Kashiwa, Chiba 277-8562; \\ ${ }^{2}$ Molecular Composite Medicine Research Group, Biomedical Research Institute, National Institute \\ of Advanced Industrial Science and Technology (AIST), Tsukuba, Ibaraki 305-8566, Japan
}

Received January 16, 2014; Accepted February 19, 2014

DOI: $10.3892 /$ br. 2014.260

\begin{abstract}
MicroRNA (miRNA) therapeutics, whereby miRNA networks are modified to treat disease, is attracting considerable attention in the field of nucleic acid therapeutics. In this study, we described highly effective constructs of miRNA-masking oligonucleotides (ONDs) that bind to mRNA and interfere with the binding of miRNA to its target site. Moreover, the correlation between ONDs and thermodynamics on the effect of masking mRNA was investigated. Using a reporter system, we evaluated ONDs of various lengths that have been linked to $O$-methyl groups and/or locked nucleic acids (LNAs) on their ability to inhibit binding of miRNA to target mRNA in cultured cells. LNAs of 10-12 nucleotides (nt) exerted the strongest inhibitory effects, which were similar to the inhibitory activity of antisense ONDs against miRNA. No such inhibitory activity was observed with the standard 22-nt $O$-methyl-modified RNA in the reporter system. The results of melting temperature experiments combined with the masking activity that prevented binding of miRNA to its target, suggested that a major contributor to the masking activity in cells may be the thermodynamic properties of the complexes. Our findings may contribute to the design of effective masking LNAs.
\end{abstract}

\section{Introduction}

MicroRNAs (miRNAs) are short [ 22 nucleotides (nt)], non-coding RNAs that regulate a variety of biological activities by adjusting gene expression via binding to their target mRNAs (1-3). As miRNAs are involved in cell differentiation, metabolism and several diseases, they have become a focus

Correspondence to: Dr Makoto Miyagishi, Molecular Composite Medicine Research Group, Biomedical Research Institute, National Institute of Advanced Industrial Science and Technology (AIST), 1-1-1 Higashi, Tsukuba, Ibaraki 305-8566, Japan

E-mail: makoto.miyagishi@aist.go.jp

Key words: locked nucleic acid, microRNA, masking of investigation in an effort to elucidate and modify these processes.

Locked nucleic acid (LNA) is an artificial moiety, which has a methylene linkage between the 2'-oxygen and the 4'-carbon of the pentose and hybridizes strongly to the complementary sequence $(4,5)$. Chemical modifications, such as 2'-fluoro, $O$-methyl and thiol-modified phosphate, are also useful techniques to improve affinity or resistance against degradation.

The strategy of miRNA masking has been used to disrupt miRNA function and involves masking the target site on target mRNA using a modified single-stranded RNA complementary to the target sequence $(6,7)$. In this study, we tested a series of miRNA-masking constructs by means of a cell reporter system and investigated the correlation between oligonucleotides (ONDs) and thermodynamics on the effect of masking mRNA.

\section{Materials and methods}

Constructs. The Renilla luciferase expression vector (pRL-SV40) and the firefly luciferase expression vector (pGL4.13) were purchased from Promega Corporation (Madison, WI, USA). The Renilla luciferase expression vector with an miRNA-21 target site (pRL-miR21) was generated by inserting an annealed OND corresponding to the target sequence of miRNA-21 into the XbaI site of pRL-SV40.

Cell culture, transfection and reporter assays. HeLa S3 cells were obtained from the Cell Bank, RIKEN BioResource Center (Tsukuba, Japan) and were grown in Dulbecco's modified Eagle's medium supplemented with $10 \%$ fetal bovine serum. Transfections were performed using Lipofectamine ${ }^{\mathrm{TM}} 2000$ reagent (Invitrogen Life Technologies, Rockville, MD, USA) according to the manufacturer's instructions. For the luciferase assays, HeLa S3 cells were cotransfected with $10 \mathrm{ng}$ of pRL-miR21, $10 \mathrm{ng}$ of pGL4.13 and the indicated amounts of ONDs in individual wells of 96-well plates. The results were normalized by reference to the firefly luciferase activity (internal control). The luciferase activity was analyzed 14-18 $\mathrm{h}$ following transfection with the dual-luciferase system (Promega Corporation). The cells were lyzed and the activities 
of firefly and Renilla luciferase were measured sequentially with a GloMax ${ }^{\circledR} 96$ Microplate Luminometer (Promega Corporation). Anti-miRNA (5'-GsAsTsAsAsGsCsT-3', where 's' indicates a phosphorothioate linkage), a bridged nucleic acid (2',4'-BNA NC) with a thiol-modified backbone against miRNA, was used as a positive control (8).

ONDs. All the modified ONDs were purchased from Gene Design, Inc., (Ibaraki, Osaka, Japan).

Measurement of melting temperatures (Tm). The Tm for the complexes between each OND and the target complementary RNA (5'-UCAACAUCAGUCUGAUAAGCUA-3') was measured with a spectrophotometer (UV-1800; Shimadzu Corporation, Kyoto, Japan) at a cooling rate of $0.5^{\circ} \mathrm{C} / \mathrm{min}$ and a temperature of $10-95^{\circ} \mathrm{C}$ in phosphate-buffered saline [40 mmol/l $\mathrm{Na}_{2} \mathrm{HPO}_{4}, 12 \mathrm{mmol} / 1 \mathrm{NaH}_{2} \mathrm{PO}_{4}$ and $154 \mathrm{mmol} / 1 \mathrm{NaCl}$ (pH 7.4)] and $2 \mathrm{mmol} / 1 \mathrm{MgCl}_{2}$. Values of $\mathrm{Tm}$ were determined from absorbance by a differential equation (9) as the temperature that corresponded to the maximum on the first derivative profile of each melting curve.

\section{Results and Discussion}

To evaluate the inhibitory effects of masking RNAs on the activity of miRNA (specifically, miRNA-21), we generated a luciferase reporter vector with an miRNA target site in the 3' untranslated region (UTR). A phosphothiol-modified BNA (anti-miRNA), which possesses an inhibitory activity similar to that of the LNA antisense against miRNA without thiol modifications, was used as a positive control. Subsequently, a standard 22-nt $O$-methyl-modified RNA (OND1 in Figs. 1 and 2) that was complementary to the target sequence of the miRNA was synthesized. The HeLa S3 cells were cotransfected with the masking RNA and Renilla luciferase reporter vector (with the target sequence of the miRNA) plus a firefly luciferase vector (internal control). The inhibitory activity was evaluated by a standard dual-luciferase assay. However, we were unable to detect an obvious masking effect of OND1 against the miRNA target at $100 \mathrm{nM}$ and lower concentrations, despite the fact that a positive control, namely the anti-miRNA antisense OND directed against miRNA-21, which bound complementarily to miRNA-21, was able to repress the effect of the miRNA (Figs. 1 and 2). An LNA was then used to enhance the activity of masking RNA. A masking OND (OND2 in Figs. 1 and 2) was synthesized with 8 LNAs at 2-nt intervals and the inhibitory activity against miRNA was assessed.

As shown in Fig. 2, we confirmed masking activity in a dose-dependent manner; however, OND2 was found to be less effective compared to the anti-miRNA antisense OND against miRNA. A previous study demonstrated that an 8-nt tiny anti-miRNA antisense LNA, which spanned the seed region of miRNA, exerted stronger inhibitory effects against miRNA compared to shorter (7-nt) or longer (9-nt) anti-miRNA LNAs (6). This finding prompted us to investigate whether an 8-nt tiny LNA may exhibit increased masking activity in preventing the binding of miRNA to its target. However, contrary to our expectations, an 8-nt masking LNA (OND3) covering the binding site of the seed region of miRNA did not exhibit any masking activity (Figs. 1 and $3 \mathrm{~A}$ ). Thus, longer

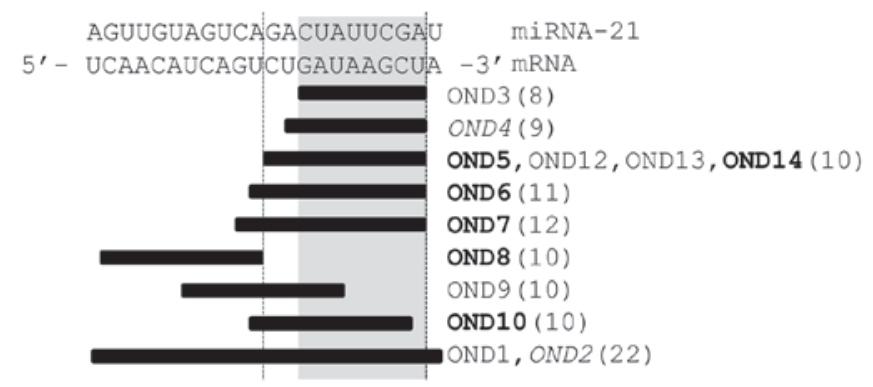

Figure 1. Design of ONDs complementary to the target site of the miRNA. The size (nt) of each OND is indicated in brackets. The ONDs in bold print exhibited strong masking activity, whereas the ONDs in italics exhibited moderate masking activity. Shadowed area, 8-nt seed match region of miRNA-21. ONDs, oligonucleotides; miRNA, microRNA; nt, nucleotides.

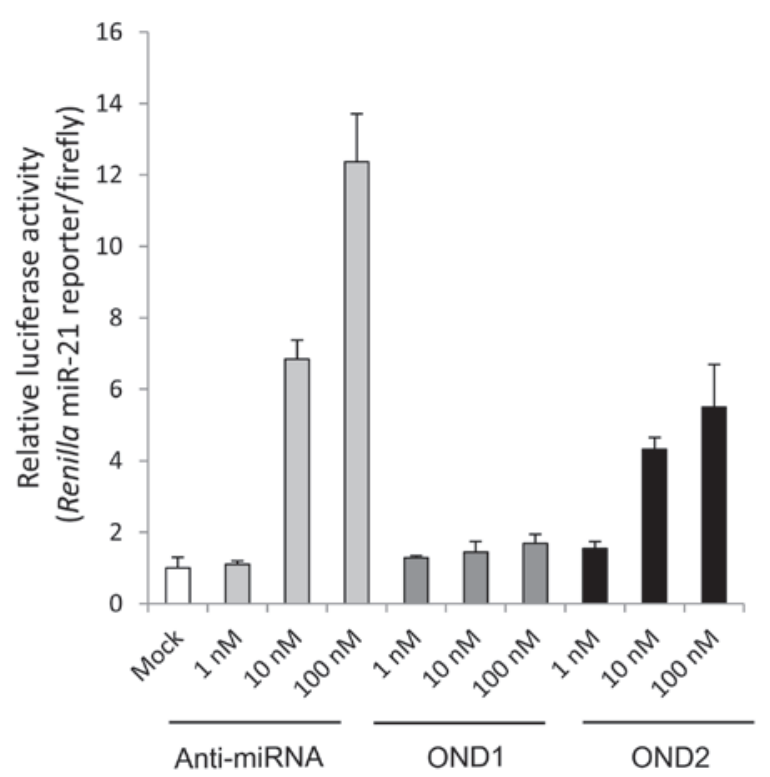

Figure 2. Conventional and current methods of masking activity in cells. The masking OND that was partially modified with a LNA (OND2) exhibited moderate activity, but the standard 22-nt $O$-methyl-modified OND (OND1) exhibited a lower masking activity in our reporter system. Data are expressed as means \pm standard deviation in triplicate. OND, oligonucleotide; LNA, locked nucleic acid; nt, nucleotides; miRNA, microRNA.

masking LNAs (OND4 through OND7; 9-12-nt, Fig. 1) that covered the binding site of the seed region of miRNA were tested for their masking activities. As shown in Fig. 3A, 10- to 12-nt masking LNAs exerted strong suppressive effects that were dose-dependent and similar to those of the positive control directed against miRNA. Judging from the relative luciferase activity of the anti-miRNA positive control, the tiny masking LNAs (10-12 nt) exhibited higher activities compared to the long LNAs (22 nt) (Figs. 2 and 3A).

The tiny 8-nt anti-miRNA antisense OND, which binds complementarily to miRNA, is specifically effective at the seed region of miRNA (6) and the activities of small interfering (si)RNAs are strongly dependent on their target sequences. Therefore, whether the activity of the tiny masking LNAs exhibit the same positional and/or sequence-related dependence (Figs. 1 and 3B) was next investigated. We designed three 10-nt tiny LNAs targeted to within the target 
A

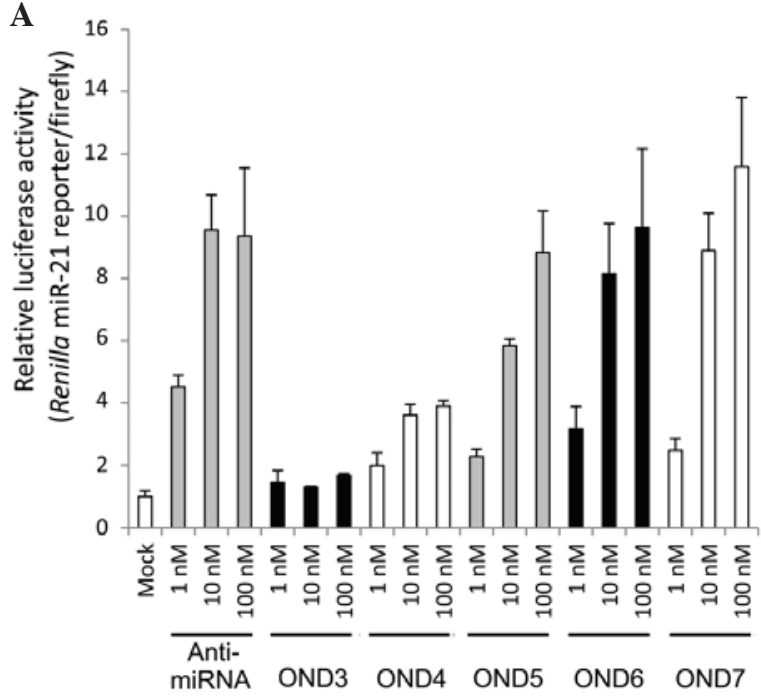

B

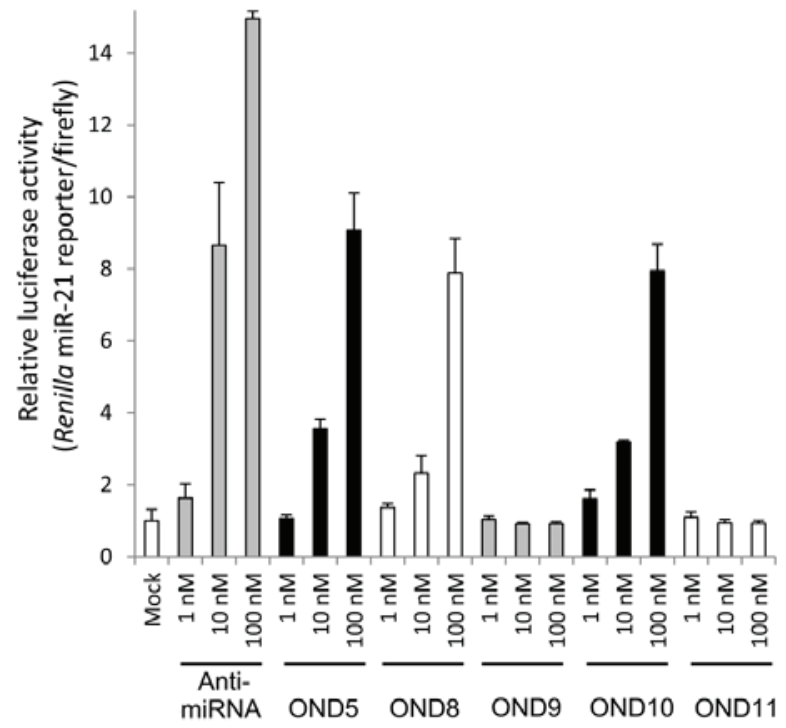

Figure 3. Evaluation of tiny LNAs. (A) The 10- to 12-nt LNAs (in OND5, OND6 and OND7), but not the 8- to 9-nt LNAs (in OND3 and OND4) exhibited strong masking activities, similar to those of the positive control antisense OND (anti-miRNA). (B) The masking activity of 10-nt ONDs depend on the position and the target sequences. OND5, OND8 and OND10 were successful in suppressing miRNA activity, while OND9 was not. OND11, which was designed to mask the site of initiation of translation within the coding region, was unsuccessful in supressing miRNA activity. Data are expressed as means \pm standard deviation in triplicate. LNA, locked nucleic acid; nt; nucleotides; OND, oligonucleotide; miRNA, microRNA.

Table I. Melting temperatures.

\begin{tabular}{lcll}
\hline Oligomers & $\begin{array}{c}\text { Length } \\
(\mathrm{mer})\end{array}$ & \multicolumn{1}{c}{ Sequences } & $\begin{array}{c}\text { Tm } \\
\left({ }^{\circ} \mathrm{C}\right)\end{array}$ \\
\hline OND1 & 22 & uagcuuaucagacugauguuga & 81.3 \\
OND2 & 22 & TagCuuAucAgaCugAugTugA & 92.4 \\
OND3 & 8 & AGCTTATC & 71.9 \\
OND4 & 9 & AGCTTATCA & 85.1 \\
OND5 & 10 & AGCTTATCAG & 92.0 \\
OND6 & 11 & AGCTTATCAGA & 92.1 \\
OND7 & 12 & TAGCTTATCAGA & 92.6 \\
OND8 & 10 & ACTGATGTTG & 90.7 \\
OND9 & 10 & ATCAGACTGA & 45.9 \\
OND10 & 10 & GCTTATCAGA & 90.2 \\
OND11 & 10 & ATCTTCCATG & n.d. \\
OND12 & 10 & AgcTtaTcaG & 73.2 \\
OND13 & 10 & agcuuaucag & 56.8 \\
OND14 & 10 & AsGsCsTsTsAsTsCsAsG & 86.2 \\
\hline
\end{tabular}

Capital letters indicate locked nucleic acids, lower case letters indicate 2'-O-methyl modification and the lower case 's' indicates a phosphorothioate linkage. OND, oligonucleotide; Tm, melting temperature; n.d., not determined.

region of the miRNA; OND8, which masks the 5 ' region of the miRNA target region (opposite the seed region) and OND10, which masks the region that extends $1 \mathrm{nt}$ upstream of OND5 (Fig. 3B). The inhibitory effects against miRNA of these tiny masking LNAs were similar to that of OND8. By contrast, OND9, which masks the central region of the miRNA target site, exerted no inhibitory effects. Notably, the masking LNAs did not appear to affect translation, even when the masking

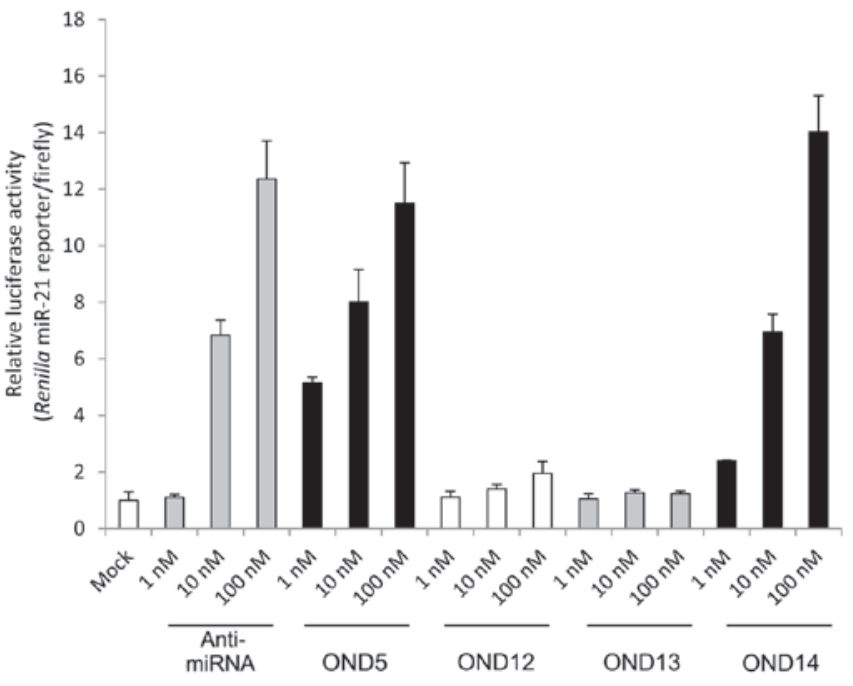

Figure 4. Comparison of the chemical modifications of 8-nt LNAs. OND12, which possesses LNA and $O$-methyl partially, and OND13, which posseses only $O$-methyl, exhibited no masking activity. OND14, which is composed of a thiol-phosphate backbone and LNA exhibited similar masking activities to that of OND5. Data are expressed as means \pm standard deviation in triplicate. nt; nucleotide; LNA, locked nucleic acid; OND, oligonucleotide.

site was within the coding region, as, for example, in the case of OND11, which was designed to mask the site of initiation of translation (Fig. 3B). It is possible that tiny LNAs act at the location where miRNAs function, but not on the ribosome (6). The precise inhibitory mechanism of the tiny LNAs requires further investigation.

To evaluate the correlation between masking activity and each masking OND in more detail, we measured the Tm of binding between masking ONDs and the complementary target RNA in vitro (Table I). The Tm of 8- to 12-nt tiny masking LNAs was closely correlated with their masking 
activities (Table I, Figs. 2 and 3A). OND9, which exhibited no masking activity in cells, yielded a low Tm, possibly as a consequence of homodimerization (Fig. 3B). OND8 and OND10, whereby the masking LNAs are directed toward the 3' region of miRNA, exhibited a high Tm that was correlated with their strong masking activities.

Taken together, our findings suggest that the masking activities of LNAs in HeLa S3 cells reflect the thermodynamic properties of the complexes between the masking LNAs and the target mRNA. To confirm our hypothesis, we generated masking OND12 and OND13, the complexes of which had a lower Tm compared to OND5, but the same sequence as OND5, and measured their masking activities against miRNA (Figs. 1 and 4). As the Tm decreased, the masking activity tended to decline, supporting our hypothesis that masking activity is dependent on the thermodynamic properties of the physical binding of the masking LNA to the target mRNA. Although the 22-nt masking OND had the same high Tm in the complex as OND9, it exhibited lower masking activity, suggesting the involvement of factors other than $\mathrm{Tm}$, such as the length of the masking OND, the stability of the OND inside the cells, the secondary structure of the mRNA and natural modification(s) at the target site.

Finally, we investigated the effects of thiol modification of the tiny LNAs on masking activity (Figs. 1 and 4). OND14 with thiol modifications of the phosphate backbone exerted the same effect as OND5. OND14, which yielded a lower Tm compared to OND5, exhibited the same masking activity as OND5 in cells, suggesting the potential effective application in vivo of our masking strategy. Thiol modification of nucleic acid linkages is required for the application of ONDs or siRNA systems in vivo, since such modifications accelerate penetration into cells and organs and enhance the stability of these nucleic acids in vivo $(6,10)$.

In this study, novel and effective masking OND constructs directed against mRNA were developed. These constructs have a similar activity to antisense OND against miRNA and higher inhibitory activity compared to conventional long masking ONDs. Our technique may be applicable for analyzing miRNA pathways and in clinical therapy. For example, the target sites of miRNA have been identified by comparing miRNA activities between reporters with a 3'UTR that includes a putative and a mutated target sequence $(11,12)$. However, tiny masking LNAs allow more direct investigation of the target sites of miRNAs. Since miRNAs regulate a large number of downstream mRNAs, their use in the clinical setting is hampered by the potential side effects when antisense ONDs are systemically applied. The miRNA-masking method, in which only specific mRNAs are masked, may lead to more specific and safer therapeutic strategies. Based on our findings, further advancement of the masking strategy may be expected, including inhibition of protein-RNA interactions, regulation of non-coding RNA networks and control of mRNA splicing.

\section{Acknowledgements}

This study was supported by internal grants (grant nos. AAZ30354Q05, AAZ30354Q06, and AAZ30354Q09) from the National Institute of Advanced Industrial Science and Technology (AIST; Tsukuba, Japan). The authors would like to thank Dr Kurita and Dr Niwa for their helpful comments and for sharing the ultraviolet spectrometer instrument; Dr Sano and Dr Nakanishi, for sharing the luminometer instrument and Ms Saeki for her technical assistance.

\section{References}

1. Filipowicz W, Bhattacharyya SN and Sonenberg N: Mechanisms of post-transcriptional regulation by microRNAs: are the answers in sight? Nat Rev Genet 9: 102-114, 2008.

2. Bartel DP: MicroRNAs: target recognition and regulatory functions. Cell 136: 215-233, 2009.

3. Ambros V: The functions of animal microRNAs. Nature 431: 350-355, 2004.

4. Obika S, Nanbu D, Hari Y, Morio K, In Y, Ishida T and Imanishi T: Synthesis of 2'-O,4'-C-methyleneuridine and -cytidine. Novel bicyclic nucleosides having a fixed $\mathrm{C}_{3}$, -endo sugar puckering. Tetrahedron Lett 38: 8735-8738, 1997.

5. Singh SK, Koshkin AA, Wengel J and Nielsen P: LNA (locked nucleic acids): synthesis and high-affinity nucleic acid recognition. Chem Commun 4: 455-456, 1998.

6. Obad S, dos Santos CO, Petri A, et al: Silencing of microRNA families by seed-targeting tiny LNAs. Nat Genet 43: 371-378, 2011.

7. Choi WY, Giraldez AJ and Schier AF: Target protectors reveal dampening and balancing of Nodal agonist and antagonist by miR-430. Science 318: 271-274, 2007.

8. Obika S, Uneda T, Sugimoto T, Nanbu D, Minami T, Doi T and Imanishi T: 2'-O,4'-C-Methylene bridged nucleic acid (2',4'-BNA): synthesis and triplex-forming properties. Bioorg Med Chem 9: 1001-1011, 2001.

9. Mergny JL and Lacroix L: Analysis of thermal melting curves. Oligonucleotides 13: 515-537, 2003.

10. Lima WF, Prakash TP, Murray HM, et al: Single-stranded siRNAs activate RNAi in animals. Cell 150: 883-894, 2012.

11. Pencheva N, Tran H, Buss C, Huh D, Drobnjak M, Busam K and Tavazoie SF: Convergent multi-miRNA targeting of ApoE drives LRP1/LRP8-dependent melanoma metastasis and angiogenesis. Cell 151: 1068-1082, 2012.

12. Iliopoulos D, Hirsch HA and Struhl K: An epigenetic switch involving NF-kappaB, Lin28, Let-7 microRNA, and IL6 links inflammation to cell transformation. Cell 139: 693-706, 2009. 